Synthesis of Natural

Products and

Potential Drugs

\title{
Synthesis of Sarpagine Alkaloids
}

\section{Key words}

alkaloids

cycloaddition

Fischer indole synthesis

ring expansion

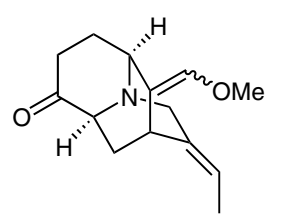

G

H-J, AcCl, MeOH, $\Delta$, then $\mathrm{H}_{2} \mathrm{O}$ Fischer indole synthesis

$\mathrm{TMSCH}_{2} \mathrm{~N}_{2}, n$-BuL $\mathrm{THF}$, then $\mathrm{MeOH}$ then silica

$80 \%$

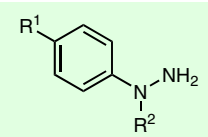

H: $R^{1}=R^{2}=H$

I: $R^{1}=H, R^{2}=M e$

J: $R^{1}=\mathrm{OMe}, \mathrm{R}^{2}=\mathrm{H}$
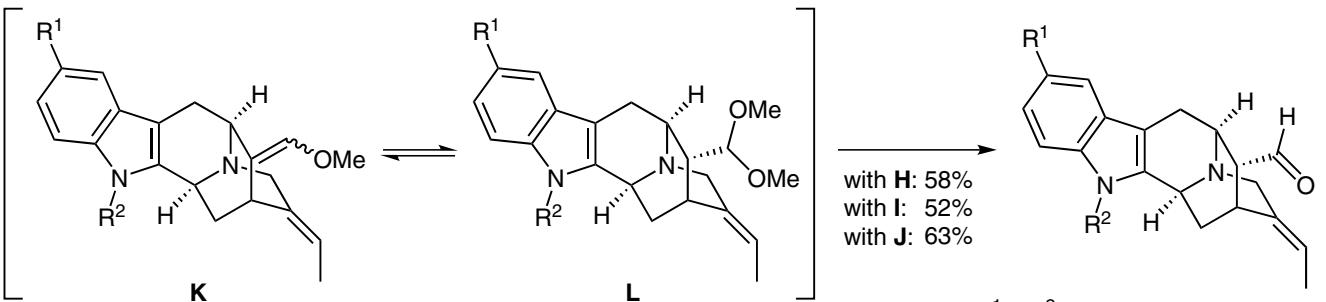

$\mathrm{R}^{1}=\mathrm{R}^{2}=\mathrm{H}:(+)$-Vellosimine

$\mathrm{R}^{1}=\mathrm{H}, \mathrm{R}^{2}=\mathrm{Me}:(+)-\mathrm{N}$-Methylvellosimine $\mathrm{R}^{1}=\mathrm{OMe}, \mathrm{R}^{2}=\mathrm{H}:(+)-10-$ Methoxyvellosimine
Significance: The authors report the enantioselective total synthesis of three sarpagine indole alkaloids which were isolated from the plant family Apocynaceae. The route relies on a common intermediate $\mathbf{G}$, which is impressively accessed using key features such as a [5+2] oxidopyridinium cycloaddition and a ring expansion. The three natural products were synthesized in only eight steps starting from known materials (12 steps from commercially available compounds).
Comment: The synthesis commenced with a [5+2] cycloaddition between oxidopyridinium salt $\mathbf{A}$ and Aggarwal's chiral ketene equivalent $\mathbf{B}$, thus yielding the desired regioisomer $\mathbf{C}$ in a 2:1 ratio. Next, ketone $\mathbf{G}$ was accessed through an intramolecular palladium-catalyzed enolate coupling of $\mathbf{D}$, followed by Wittig reaction, deprotection of the dithiolane, and ring expansion. The indole was introduced in the last step by a Fischer indole synthesis using phenylhydrazines with different substitution patterns to afford the three targets. 